\title{
N92-22060
}

\section{QUANTUM SPATIAL PROPAGATION OF SQUEEZED LIGHT IN A DEGENERATE PARAMETRIC AMPLIFIER*}

\author{
Ivan H. Deutsch \\ Department of Physics, University of California Berkeley, California, 94720 \\ John C. Garrison
}

Lawrence Livermore National Laboratory, University of California, Livermore ,, California 94550

\begin{abstract}
Differential equations which describe the steady state spatial evolution of nonclassical light are established using standard quantum field theoretic techniques. A Schrodinger equation for the state vector of the optical field is derived using the quantum analog of the slowly varying envelope approximation (SVEA). The steady state solutions are those that satisfy the time independent Schrodinger equation. The resulting eigenvalue problem then leads to the spatial propagation equations. For the degenerate parametric amplifier this method shows that the squeezed state is the ground state of the squeezing Hamiltonian. The magnitude and phase of the squeezing parameter obey nonlinear differential equations coupled by the amplifier gain constant and phase mismatch. The solution to these differential equations is equivalent to one obtained from the classical three wave mixing steady state solution to the parametric amplifier with a nondepleted pump.
\end{abstract}

\section{Introduction}

The standard approach in quantum optics for dealing with nonclassical light is to introduce a normal mode expansion for the field. This naturally leads to time evolution equations for the mode amplitudes (creation and annihilation operators) which satisfy the canonical equal time commutation relations. Such an approach is well suited for dealing with systems in optical cavities, such as an optical parametric oscillator, but is not appropriate for open systems such as a parametric amplifier. For the amplifier we are usually interested in the spatial dependence of temporally steady state fields. This problem has been treated for quantum fields either by identifying spatial evolution with temporal evolution for an interaction time set by the effective interaction length [1-4], or through the use of new quantization methods which require nonconventional commutation relations that are generally inconsistent with the fundamental equal time commutators for interacting fields [5-9]. In addition, the problem has recently been studied using the positive-P representation and stochastic differential equations [10]. In this letter we propose an alternative scheme which employs only standard quantum field theoretic techniques, and apply this analysis to the degenerate parametric amplifier (DPA) in 
order to study the spatial evolution of squeezed light.

In the classical three wave mixing analysis of the DPA, the positive frequency component of the electric field is written as a slowly varying time independent envelope multiplying a carrier plane wave. Such an ansatz physically represents steady state propagation and leads to spatial differential equations for the envelope. Quantum mechanically, this ansatz cannot hold as an operator identity in the Heisenberg picture. Upon closer inspection, we see that the requirement of steady state propagation is enforced through the choice of state vector for the field. More precisely, steady state fields are the stationary states of the appropriate Hamiltonian. The resulting eigenvalue problem is then a time independent Schrodinger equation, and leads to spatial differential equations for the functions which parameterize the global state vector. We will apply this technique to the DPA in one dimension with a classical nondepleted pump. The stationary state solutions are squeezed states specified by a set of coupled nonlinear differential equations for the magnitude and phase of the squeezing parameter driven by the coupling constant of the three wave mixing.

\section{Steady State Propagation Condition}

Using the classical wave mixing analysis as our guide, we formulate the quantum theory in terms of the SVEA. The positive frequency electric field operator is written as an envelope field modulating a carrier plane wave of a given polarization $\boldsymbol{\varepsilon}$. For systems in which the medium has no transverse dependence over the beam diameter and is longitudinally short compared to the Raleigh range, the theory becomes essentially one dimensional so that,

$$
\mathbf{E}_{\omega}^{(+)}(\mathbf{x}, \mathrm{t})=\varepsilon \sqrt{\frac{2 \pi \hbar \omega}{\mathrm{An}^{2}(\omega)}} \Psi(z, t) \mathrm{e}^{\mathrm{i}(\mathrm{kz}-\omega \mathrm{t})}
$$

where $A$ is the beam area, and $n(\omega)$ is the linear index of refraction. The effective field theory in the SVEA formally resembles a nonrelativistic many body theory for a complex scalar field $\Psi$ [12],

$$
\left[\Psi(\mathrm{z}, \mathrm{t}), \Psi^{\dagger}\left(\mathrm{z}^{\prime}, \mathrm{t}\right)\right]=\delta\left(\mathrm{z}-\mathrm{z}^{\prime}\right)
$$

The Hamiltonian (in the Heisenberg picture) can be written as,

$$
\begin{aligned}
& \mathrm{H}=\mathrm{H}_{\mathrm{c}}+\mathrm{H}_{\mathrm{env}}+\mathrm{H}_{\mathrm{int}} \\
& \mathrm{H}_{\mathrm{c}}=\hbar \omega \int \mathrm{d}^{3} \mathrm{x} \Psi^{\dagger} \Psi
\end{aligned}
$$




$$
\begin{aligned}
& H_{\text {env }}=\frac{-i \hbar}{2} \frac{c}{n(\omega)} \int \mathrm{dz}\left(\Psi^{\dagger} \frac{\partial \Psi}{\partial z}-\frac{\partial \Psi^{\dagger}}{\partial z} \Psi\right) \\
& H_{\text {int }}=\frac{i \hbar}{2} \frac{c}{n(\omega)} \int \mathrm{dz}\left(\kappa^{*}(z) \mathrm{e}^{-i \omega_{\mathrm{p}} t} \Psi^{2}(\mathrm{z}, \mathrm{t})-\kappa(\mathrm{z}) \mathrm{e}^{\left.\mathrm{i} \omega_{\mathrm{p}} \mathrm{t} \Psi^{\dagger 2}(\mathrm{z}, \mathrm{t})\right)}\right.
\end{aligned}
$$

where we have decomposed the "free" field Hamiltonian into the carrier wave Hamiltonian $\mathrm{H}_{\mathrm{c}}$, and the Hamiltonian governing the free (plus linear) evolution of the envelope $\mathrm{H}_{\text {env }}$. The effective interaction Hamiltonian for the DPA is given by $\mathrm{H}_{\text {int }}[14]$ representing the mixing through the nonlinear susceptibility, with pump frequency $\omega_{\mathrm{p}}$. The interaction coupling is determined by

$$
\begin{aligned}
& \kappa(z)=\frac{1}{2} g_{0}(z) e^{i \phi(z)} \\
& g_{0}(z)=\frac{4 \pi \omega}{n(\omega) c}\left|\chi^{(2)}(z)\right|\left|\mathcal{E}_{P}(z)\right| \\
& \phi(z)=-\frac{\pi}{2}+\Delta k z+\vartheta(z)
\end{aligned}
$$

where $g_{0}(z)$ is the standard power gain coupling constant [13] (allowing for possible $z$-dependence), $\Delta \mathrm{k}$ is the phase mismatch at the degenerate frequency, and $\vartheta(z)$ is the remaining phase stemming from the pump and $\chi^{(2)}$.

We seek the quantum field state vector corresponding to steady state propagation. Maintaining our close analogy with the classical analysis, we remove the carrier wave oscillation from the dynamics of this state. Quantum mechanically we use the envelope picture (E.P.) originally introduced by Caves [11], in which the dynamics of the operators are dictated by the carrier wave Hamiltonian, and the states evolve by a Schrodinger equation with the Hamiltonian $\mathrm{H}_{\mathrm{env}}+\mathrm{H}_{\mathrm{int}}$. If we denote objects in this picture by the superscript $(E)$, then the dynamical equations in this picture are

$$
\begin{aligned}
& i \hbar \frac{\partial A^{(E)}}{\partial t}=\left[A^{(E)}, H_{c}^{(E)}\right] \\
& i \hbar \frac{\partial|\Phi\rangle^{(E)}}{\partial t}=\left(H_{e n v}^{(E)}+H_{i n t}^{(E)}\right)|\Phi\rangle^{(E)} .
\end{aligned}
$$

For steady-state applications there will be exact frequency matching between the carrier frequencies of the various waves which mix through the nonlinear interaction, so that the Hamiltonian in Eq. (5b) will be independent of time. The steady state (ss) solutions are thus identified with the stationary solutions to Eq. (5b),

$$
\left(H_{\mathrm{env}}^{(\mathrm{E})}+\mathrm{H}_{\mathrm{int}}^{(\mathrm{E})}\right)|\Phi\rangle_{\mathrm{ss}}^{(\mathrm{E})}=\lambda|\Phi\rangle_{\mathrm{ss}}^{(\mathrm{E})}
$$


Henceforth, the labels (E) and (ss) will be omitted. Eq. (6) is the key result of our analysis which we now apply to the parametric amplifier.

\section{Propagation Solution to the DPA}

It is well known that the output produced during a parametric interaction is a squeezed vacuum state [11]. In an oscillator configuration one can restrict attention solely to a single mode of the cavity (for a doubly degenerate oscillator). In the amplifier configuration under consideration here, we must generalize the squeezing operator to take into account the many modes associated with the envelope field. We define a functional squeezing operator,

$$
\mathrm{S}[\zeta] \equiv \exp \left\{\frac{1}{2} \int \mathrm{dz}\left(\zeta^{*}(\mathrm{z}) \Psi^{2}(\mathrm{z})-\zeta(\mathrm{z}) \Psi^{\dagger 2}(\mathrm{z})\right)\right\}
$$

with z-dependent squeezing parameter $\zeta(z)=r(z) e^{i \theta(z)}$. One can easily show that the Bogoliubov transformation generalizes to

$$
\widetilde{\Psi}(z) \equiv S^{\dagger}[\zeta] \Psi(z) S[\zeta]=\cosh (r(z)) \Psi(z)-e^{i \theta(z)} \sinh (r(z)) \Psi^{\dagger}(z)
$$

The squeezed vacuum,

$$
|\{\zeta\}\rangle \equiv S[\zeta]|0\rangle
$$

satisfies the well known eigenvalue equation [15],

$$
\begin{aligned}
& \widehat{\Psi}(\mathrm{z})|\{\zeta\}\rangle=0 \\
& \widehat{\Psi}(\mathrm{z}) \equiv \mathrm{S}[\zeta] \Psi(\mathrm{z}) \mathrm{S}^{\dagger}[\zeta]=\cosh (\mathrm{r}(\mathrm{z})) \Psi(\mathrm{z})+\mathrm{e}^{\mathrm{i} \theta(\mathrm{z})} \sinh (\mathrm{r}(\mathrm{z})) \Psi^{\dagger}(\mathrm{z})
\end{aligned}
$$

Using the formalism developed in Section 2, we deduce the steady steady solutions by solving the time independent Schrodinger equation (6). We take as our ansatz for the eigenvector a squeezed vacuum state Eq. (9), i.e.

$$
\left(\mathrm{H}_{\mathrm{env}}+\mathrm{H}_{\mathrm{int}}\right)|\{\zeta\}\rangle=\lambda|\{\zeta\}\rangle
$$

Our goal is to prove Eq. (11) and thereby obtain differential equations for the spatial squeezing 
parameter $\zeta(\mathrm{z})$. Together, Eqs. (10a) and (11) imply

$$
\left[\mathrm{H}_{\mathrm{env}}+\mathrm{H}_{\mathrm{int}}, \widehat{\Psi}(\mathrm{z})\right]|\{\zeta\}\rangle=0
$$

Substituting Eqs. (3) and (10b) into Eq. (12), using Eq. (8), and the relation (4a) for the polar decomposition of $\mathrm{K}$ gives,

$$
\left[\left(\frac{d r}{d z}-\frac{1}{2} g_{0} \cos (\theta-\phi)\right)+\frac{i}{2}\left(\frac{d \theta}{d z} \sinh (2 r)+g_{0} \cosh (2 r) \sin (\theta-\phi)\right)\right] \Psi^{\dagger}|0\rangle=0 .
$$

Thus, the eigenvalue condition requires the real and imaginary parts of the coefficient of $\Psi^{\dagger}$ to vanish, yielding the desired propagation equations:

$$
\begin{aligned}
& \frac{d r}{d z}=\frac{1}{2} g_{0} \cos (\theta-\phi) \\
& \frac{d \theta}{d z}=-g_{0} \operatorname{coth}(2 r) \sin (\theta-\phi) .
\end{aligned}
$$

The solution to (14) produces a relation for the squeezing parameters $\{r(z), \theta(z)\}$ in terms of the experimental parameters $\left\{g_{0}(z), \phi(z)\right\}$. In the next section we give an interpretation of the meaning of these equations and analyze their solutions.

\section{Solutions and Interpretation of Propagation Equations}

In solving Eqs. (14) we take as our boundary conditions,

$$
\begin{aligned}
& r(0)=0 \\
& \theta(0)=\phi(0)
\end{aligned}
$$

to ensure the continuity of the squeezing parameter. In order to get a better understanding of the nature of the equations we examine them for various regimes. Consider first the situation in which the phase of $\kappa, E q .(4 c)$, is a constant $\phi(z)=\phi_{0}$ (i.e exact phase matching). With boundary conditions (15), the solution to (14) is immediately seen to be

$$
\begin{aligned}
& r(z)=\int_{0}^{z} d z^{\prime} \frac{1}{2} g_{0}\left(z^{\prime}\right), \\
& \theta(z)=\phi_{0}
\end{aligned}
$$


For a uniform crystal with plane wave pump, this yields the expected result $r_{\text {out }}=\frac{1}{2} g_{0} L$ (for $z \geq L$ ), where $\mathrm{L}$ is the length of the crystal.

In limit of short crystals, Eqs. (14) can be expanded for small $\mathrm{r}$ to yield

$$
\left\{\begin{array}{l}
\frac{\mathrm{dr}}{\mathrm{dz}}=\frac{1}{2} \mathrm{~g}_{0} \cos (\theta-\phi) \\
\frac{\mathrm{d} \theta}{\mathrm{d} z}=-\frac{\mathrm{g}_{0}}{2 \mathrm{r}} \sin (\theta-\phi)
\end{array}\right\} \Rightarrow \frac{\partial \zeta}{\partial \mathrm{z}}=\kappa(\mathrm{z})
$$

Thus, in this limit the complex squeezing parameter is just the integral of the interaction coupling constant. Henceforth we consider crystals with uniform nonlinearity and a plane wave pump, so that $\mathrm{g}_{0}$ is a constant and $\phi(z)=-\frac{\pi}{2}+\Delta \mathrm{kz}$. The solution to Eq. (17) for $z \geq L$ is of a familiar form,

$$
\begin{aligned}
& r_{\text {out }}=\frac{1}{2} g_{0} L \frac{\sin (\Delta \mathrm{kL} / 2)}{(\Delta \mathrm{kL} / 2)} \\
& \theta_{\text {out }}=-\frac{\pi}{2}+\Delta \mathrm{kL} / 2 .
\end{aligned}
$$

If in addition $\Delta \mathrm{k}<<1 / \mathrm{L}$ the amplitude and phase essentially decouple. However, as the phase mismatch increases, the magnitude of the squeezing decreases. Physically, large phase mismatch would cause squeezing along wildly different quadratures as the beam propagates through the crystal, thereby degrading the net squeezing.

For crystals containing many gain lengths, we expect strong sensitivity to the phase mismatch since the nonlinear coupling between $\mathrm{r}$ and $\theta$ causes rapid oscillations that destroy the magnitude of the squeezing at some value of $\Delta \mathrm{k}$. To see this we numerically integrate Eq. (14). In presenting the data, we measure all distances in units of the natural length scale $1 / \mathrm{g}_{0}$. Fig.(1) shows the magnitude of the squeezing parameter as a function of $z$ for various phase mismatches. Fig. (2) shows the squeezing parameter of downconverted light as function of phase mismatch for various values of the crystal length L, plotted simultaneously with the small r expression, Eq. (18a). For short lengths, Eq. (18a) is an excellent approximation. However, for crystals many gain lengths long (as can be obtained using intense pumps [16]) we see a sharp cutoff in the squeezing at the critical value $\Delta \mathrm{k} / \mathrm{g}_{0}$ $=1$. The sharp transition in output when $\Delta \mathrm{k}=\mathrm{g}_{0}$ is also characteristic of the classical three wave mixing solution to the parametric amplifier. This leads us to hypothesize that the solution to Eqs. (14) can be obtained from the classical propagation equations.

The classical solution for the envelope in a DPA is [13]

$$
A(z)=\left(\mu(z) A(0)+i v(z) A^{*}(0)\right) e^{i \Delta k z / 2}
$$




$$
\begin{aligned}
& \mu(z)=\cosh \left(\frac{1}{2} g z\right)-i \frac{\Delta k}{g} \sinh \left(\frac{1}{2} g z\right) ; v(z)=\sqrt{1-|\mu(z)|^{2}}=\frac{g_{0}}{g} \sinh \left(\frac{1}{2} g z\right) \\
& g=\sqrt{g_{0}^{2}-(\Delta k)^{2}} .
\end{aligned}
$$

If we replace the c-number fields with creation/annihilation operators $A^{*}(0) \rightarrow a^{\dagger}, A(z) \rightarrow b$ representing "in" and "out" fields respectively, we recover the solution obtained by Caves and Crouch [5], and Huttner et. al. [6],

$$
b=\left(\mu(z) a+i v(z) a^{\dagger}\right) e^{i \Delta k z / 2}
$$

The unitary operator relating a to $b, b=U^{\dagger} a U$, is a generalized squeezing operator

$$
\mathrm{U}=\mathrm{R}^{\dagger}\left(\frac{1}{2}(\operatorname{Arg}(\mu)+\Delta \mathrm{kz})\right) S\left(-\sinh ^{-1}(v)\right)
$$

where $S$ is the standard squeezing operator and $R$ is the phase rotation operator [11]. This unitary operator transforms the vacuum into a squeezed state with squeezing parameter $\zeta=\mathrm{re}^{\mathrm{i} \theta}$ given by,

$$
\begin{aligned}
& r(z)=\sinh ^{-1}(v(z)) \\
& \theta(z)=-\frac{\pi}{2}+\operatorname{Arg}(\mu(z))+\Delta k z
\end{aligned}
$$

Figs. (1) and (2) are now easily understood in terms of limiting cases of (22a). In fact, direct substitution reveals that (22) is indeed the analytic solution to Eq. (14).

To understand the meaning of this result consider the nature of the interaction more carefully. Because we have assumed a classical nondepleted pump, the propagation equations are linear in the signal and idler amplitudes. In such a situation one expects the q-number and c-number solutions to be equivalent, i.e., the quantum fluctuations should propagate like a classical signal. This provides a substantial test on our formalism. The successful use of nonconventional commutation relations by previous workers is explained by a combination of good physical intuition and the special linear character of this problem. Indeed, Huttner et. al. showed that for this particular interaction the equal-z commutation relations are consistent with the fundamental equal-t commutators. However, for a general interaction in which the wave mixing equations are truly nonlinear we expect this method to fail. For example, if the pump is treated as a quantum mechanical field, then we find that the equal-z commutation relations are no longer consistent with the fundamental equal-t commutators. Thus, for more complex systems there will be qualitative differences between the classical and quantum propagation solutions. The formalism we have presented here is sufficiently general to handle these situations. 


\section{Acknowledgements:}

The authors would like to thank Aephraim Steinberg, Paul Kwiat, and Raymond Chiao for many useful conversations. I. H. D. acknowledges support by a Department of Education Fellowship.

\section{References}

* This research was performed under the auspices of the U. S. Department of Energy at the Lawrence Livermore National Laboratory under contract no. W-7405-Eng-48.

1. Shen,Y.R., 1967, Phys Rev. 155, p. 921.

2. Tucker, J., and Walls, D.F., 1969, Phys. Rev. 178, p. 2036.

3. Lane, A., Tombesi, P., Carmichael, H.J., and Walls, D.F., 1983, Opt. Com. 48, p.155.

4. Hong, C.K., and Mandel, L., 1985, Phys. Reav A 31, p. 2409.

5. Caves, C. M., and Crouch, D.D., 1987, J. Opt. Soc. Am. B 4, p. 1535.

6. Huttner, B., Serulnik, S., and Ben-Aryeh, Y., 1990, Phys. Rev. A 42, p. 5594.

7. Imoto, N. in Proceedings of the $3^{\text {rd }}$ International Symposium on the Foundations of Quantum Mechanics, Tokyo, 1989, pp. 306-314

8. Yurke, B., Grangier, P., Slusher, R.E., and Potasek, M.J., 1987, Phys. Rev. A 35, p. 3586.

9. Potesek, M. J., and Yurke, B., 1987, Phys. Rev. A 35, p. 3974.

10. Drummond, P.D., and Carter, S.J., 1987, J. Opt. Soc. Am. B4,1565, p. 6845.; Raymer, M.G., Drummond, P.D., and Carter, S.J., 1991, (unpublished).

11. Caves, C. M. and Schumaker, B. L., 1985 Phys. Rev. A 31, p. 3068.

12. Deutsch, I.H. and Garrison, J.C., 1991 Phys. Rev. A 43, p. 2498.

13. Yariv, A., 1989, Quantum Electronics, 3rd Ed.John Wiley and Sons, Inc., New York.

14. Hillery, M., and Mlodinow, L., 1984 Phys. Rev. A 30, p. 1860.

15. Yuen, H., 1976, Phys. Rev. A, 13, p. 2226.

16. Slusher, R. E., Grangier, P., A LaPorta, Yurke, B., and Potasek, M. J., 1987, Phys. Lett. 59, p. 2566. 

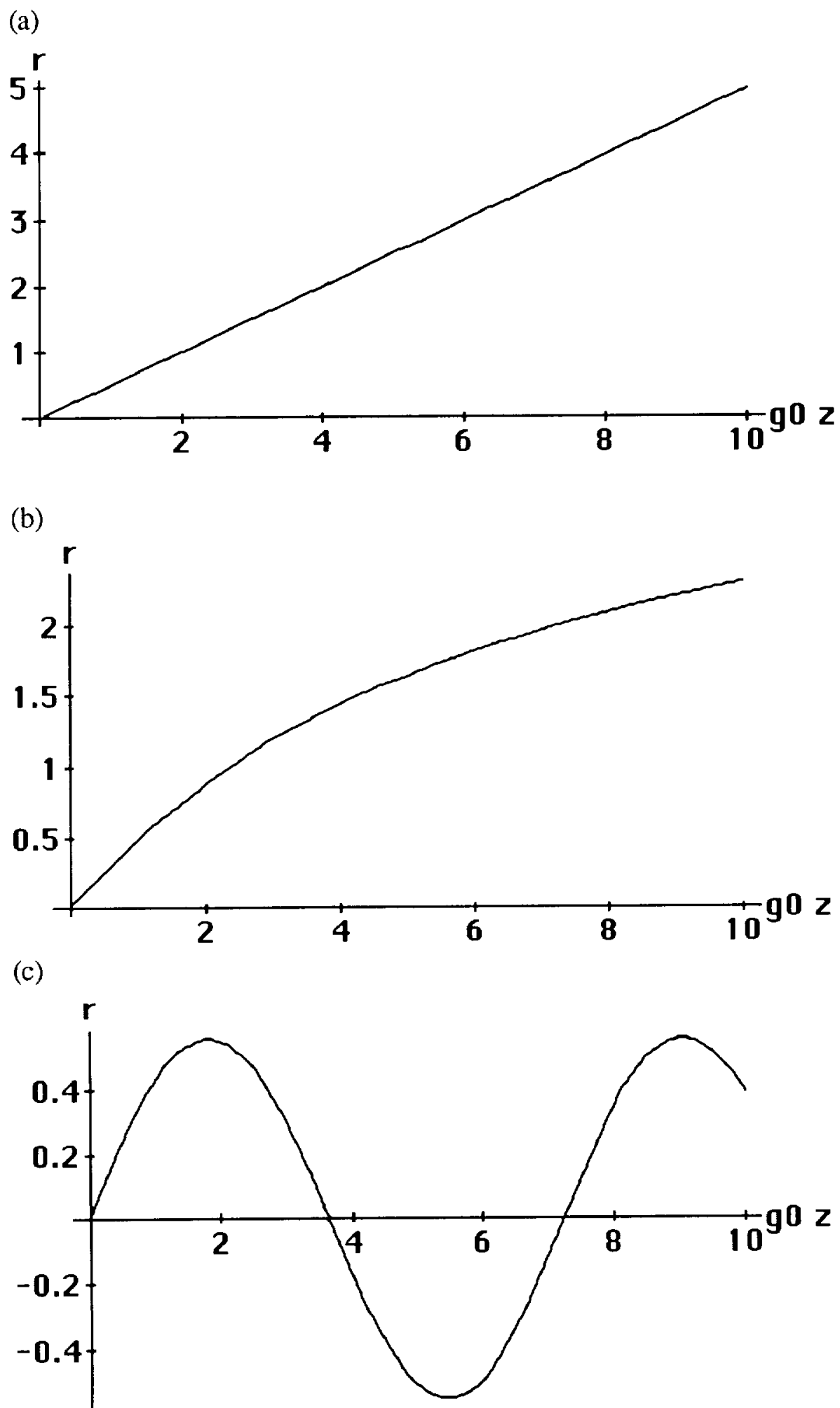

Fig 1. Squeezing magnitude as a function of propagation distance $\mathrm{z}$ measured in gain lengths $\mathrm{g}_{0}$, for different values of phase mismatch $\Delta \mathrm{k}$ : (a) $\Delta \mathrm{k}=0.1 \mathrm{~g}_{0}$, (b) $\Delta \mathrm{k}=\mathrm{g}_{0}$, (c) $\Delta \mathrm{k}=2 \mathrm{~g}_{0}$. 
(a)

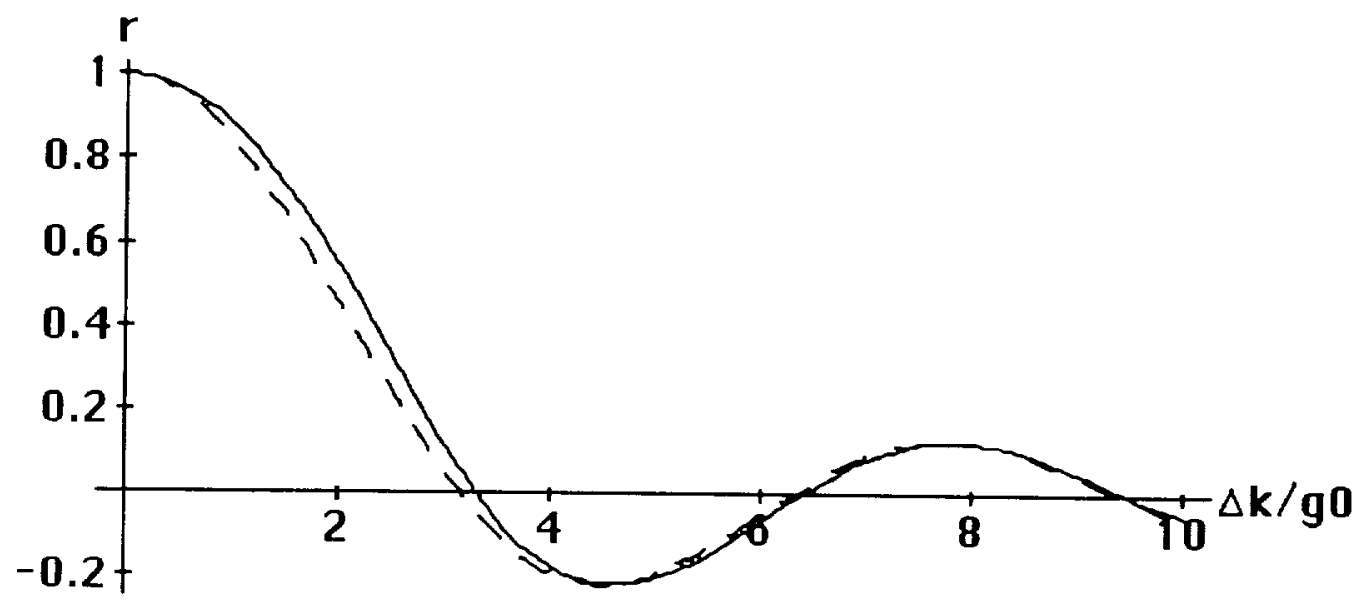

(b)

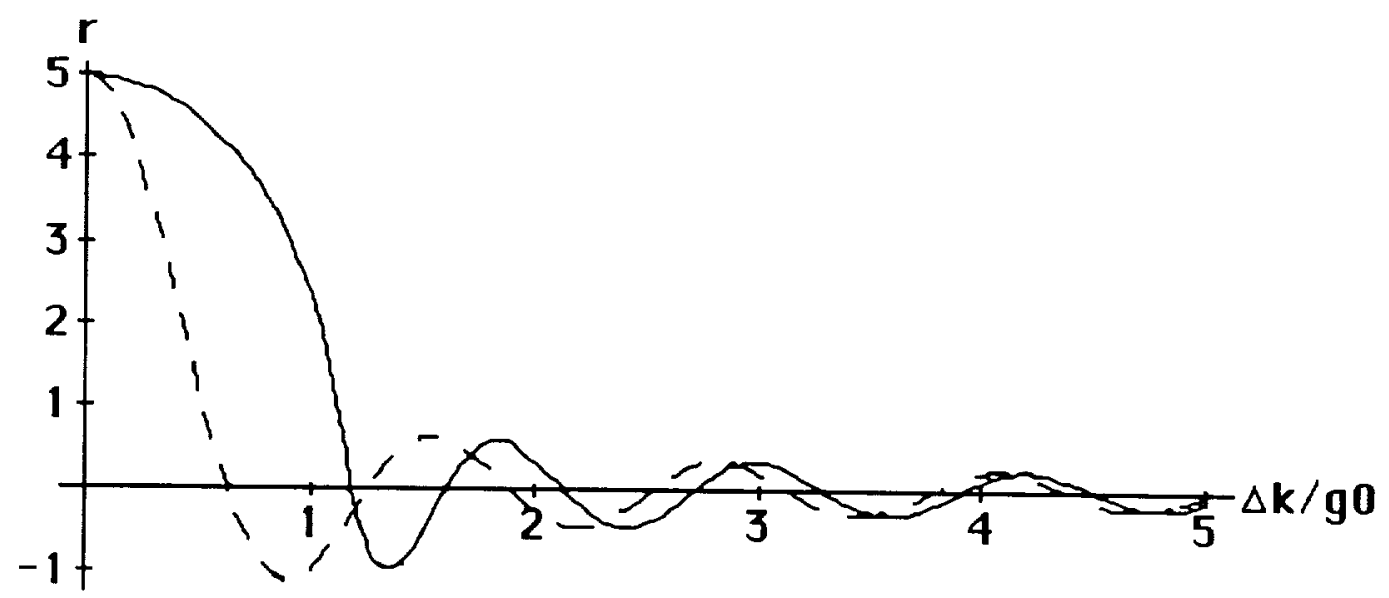

(c)

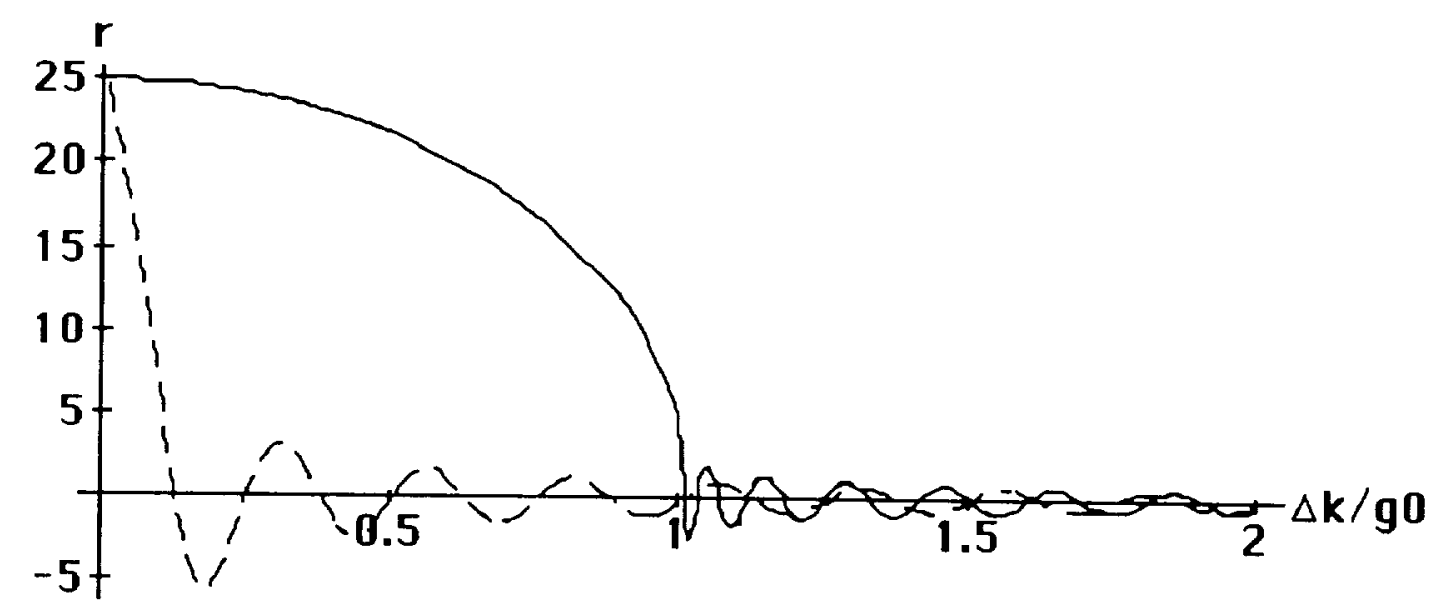

Fig 2. Squeezing at the output face as a function of $\Delta \mathrm{k}$ : (a) $\mathrm{L}=2 \mathrm{~g}_{0}$, (b) $\mathrm{L}=10 \mathrm{~g}_{0}$, (c) $\mathrm{L}=50 \mathrm{~g}_{0}$. The solid curve is the solution to Eq. (14), the dotted curve is given by the asymptotic result Eq. (18a). 